\title{
$\underline{\text { Hyperpositive non-linear effects: enantiodivergence and modelling }}$
}

\author{
Yannick Geiger, Thierry Achard, Aline Maisse-François and Stéphane Bellemin-Laponnaz* \\ Institut de Physique et Chimie des Matériaux de Strasbourg, Université de Strasbourg-CNRS \\ UMR 7504, Strasbourg, France. \\ e-mail: bellemin@unistra.fr
}

The chiral ligand N-methylephedrine (NME) was found to catalyse the addition of dimethylzinc to benzaldehyde in an enantiodivergent way, with a monomeric and a homochiral dimeric complex both catalysing the reaction at a steady state and giving opposite product enantiomers. A change in the sign of the enantiomeric product was thus possible by simply varying the catalyst loading or the ligand ee, giving rise to an enantiodivergent non-linear effect. Simulations using a mathematical model confirmed the possibility of such behaviour and showed that this can lead to situations where a reaction gives racemic products, although the system is composed only of highly enantioselective individual catalysts. Furthermore, depending on the dimer's degree of participation in the catalytic conversion, enantiodivergence may or may not be observed experimentally, which raises questions about the possibility of enantiodivergence in other monomer/dimer-catalysed systems. Simulations of the reaction kinetics showed that the observed kinetic constant $k_{\text {obs }}$ is highly dependent on user-controlled parameters, such as the catalyst concentration and the ligand ee, and may thus vary in a distinct way from one experimental setup to another. This unusual dependency of $k_{\text {obs }}$ allowed to confirm that a previously observed U-shaped catalyst order vs. catalyst loading-plot is linked to the simultaneous catalytic activity of both monomeric and dimeric complexes.

\section{Introduction}

Non-linear effects (NLEs) in asymmetric catalysis refer to cases in which the enantiomeric excess of the product does not scale linearly with the enantiomeric excess of the catalyst. ${ }^{1}$ The first examples and models of such behavioural differences between scalemic and enantiomerically pure catalysts were established by Kagan in $1986 .{ }^{2}$ Since then NLEs are considered as ubiquitous phenomena that provide additional information regarding the aggregation state of the catalyst or the formation of multiligand species. ${ }^{2-5}$ Not only being indicative of the catalytic system, NLEs also give clues to discussions on the origin of molecular homochirality in biology which is related to the origin of life. ${ }^{6}$ 
Several models for NLEs have been described and discussed in the literature, all of them being the results of interactions between the enantiomers of the chiral catalyst thus generating diastereomeric perturbations of the entire system. A positive non-linear effect (i.e. asymmetric amplification, (+)-NLE) is essentially generated by the presence of a reservoir of racemic ideally catalytically inactive hetero-aggregate (meso), ${ }^{7-9}$ although pure homochiral aggregation can also lead to (+)-NLEs in certain cases. ${ }^{10}$ Amongst these models, Kagan established a hypothetical case on where an unprecedented phenomenon could occur - that is the chiral catalyst [would] be much more efficient when partially resolved than when enantiomerically pure. We recently have observed such a case, known as hyperpositive NLE, in the enantioselective addition of dialkylzincs to benzaldehyde when catalysed by the chiral N-benzylephedrine (NBE) ligand. ${ }^{11,12}$ Subsequent mechanistic investigations pointed towards a two-component catalysis where monomeric as well as homochiral dimeric catalysts are in equilibrium and in competition: both catalyse the reaction with different enantioselectivities, the dimeric catalyst being the less enantioselective one (Figure 1). Through the precipitation of a heterochiral aggregate, variation of the ligand ee leads to a change of the overall catalyst concentration and, therefore, to a change of the monomer-dimer equilibrium. This favours the more enantioselective monomeric catalyst at low ligand ee and gives rise to the hyperpositive non-linear effect (Figure 2a, orange crosses). These findings challenge the widely applied Noyori model for asymmetric dialkylzinc additions, where only monomers are catalytically active, and shows how complex systems with concurrent catalytic cycles can emerge from a minimum of components. ${ }^{9,13}$

In line with our studies on NLEs, we have explored additional ephedrine-based ligands in dialkylzinc addition reactions. The results presented in this work show that $N$-methylephedrine ligand (NME) follows the same model as the related NBE ligand, with monomer and dimer both catalysing the reaction, the only difference being that no meso aggregate precipitates in case of NME. However, the homochiral dimer catalyst not only yields the product with a lower ee than the monomer catalyst, it selectively gives the opposite enantiomeric product. This enantiodivergent behaviour allowed us to switch between the preference for either enantiomer of the product, just by varying the catalyst loading. Variation of the enantiomeric excess of the ligand also allowed to switch the selectivity of the product formation, leading to an enantiodivergent non-linear effect. In addition, we developed a theoretical model giving a closed mathematical expression which takes into account the concomitant catalysis by monomeric and dimeric species. From this we simulated [product ee vs catalyst concentration] and [product ee vs ligand ee]-plots which allowed us to gain a better understanding of the two-component catalytic system. Furthermore, the model allowed us also to analyse the reaction kinetics. Simulations showed that the observed kinetic constant of 
the system $\mathrm{k}_{\mathrm{obs}}$ is indeed not unique for each catalytic system, but depends on user-controlled parameters such as the total catalyst concentration and the ligand ee. This has confirmed the hypotheses made earlier and allowed the simulation of a U-shaped catalyst order vs catalystcurve, which had been previously observed experimentally in the NBE-catalysed reaction. ${ }^{11}$

The paper is organised as follows: we will 1) first present the experimental results obtained from the NME ligand, showing enantiodivergence in product ee (ee $\left.e_{\mathrm{P}}\right)$ vs ligand ee $\left(\mathrm{ee}_{\mathrm{L}}\right)$ - and ee vs. catalyst loading-plots, 2) then go on to a theoretical analysis where we present the mathematical model and discuss ee $e_{P}$ vs catalyst concentration- and $e_{P}$ vs $e_{L}$-plots, 3 ) this is followed by a kinetic analysis of the system in which we investigate the relation of $\mathrm{k}_{\text {obs }}$ with the catalyst concentration and $e_{\mathrm{L}}$ and simulate catalyst order vs catalyst loading-plots. 4) Finally, we will discuss the impact of the simulations in a more general way and compare them with the experimental results.

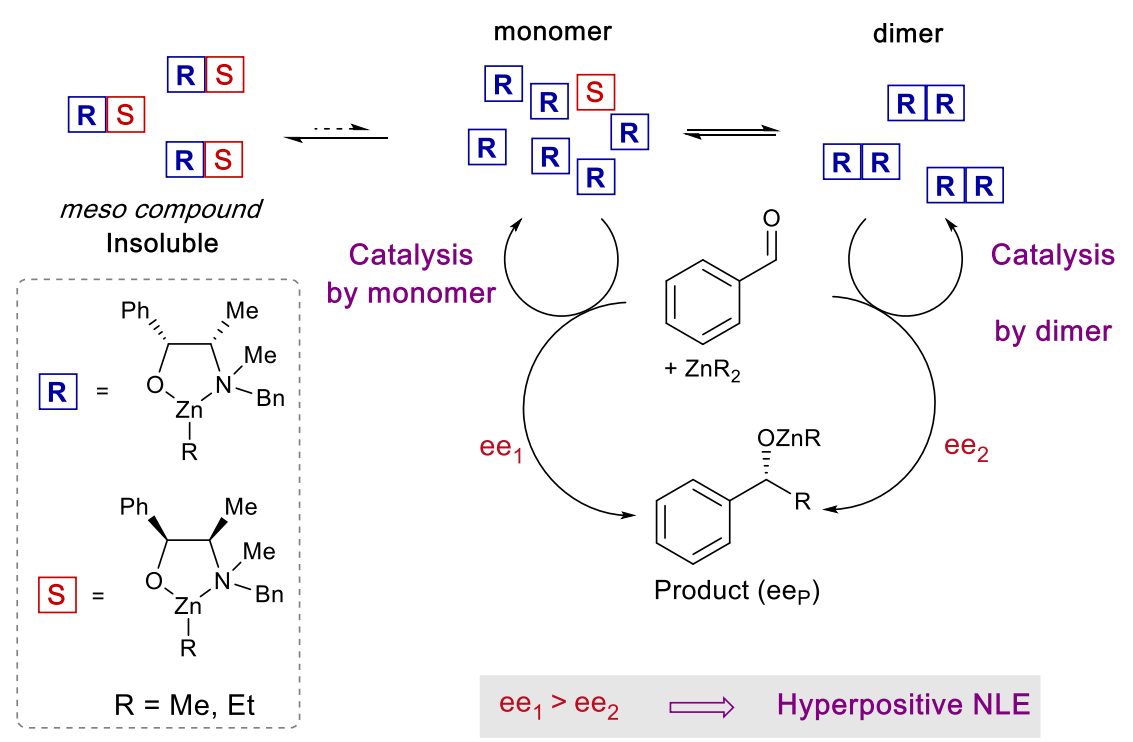

Figure 1. Competitive reaction pathway of a monomeric and a dimeric catalyst in an enantioselective reaction that account for an hyperpositive non-linear effect: the case of NBEcatalysed enantioselective addition of dialkylzinc to benzaldehyde. 


\section{Results \& Discussion}

\section{Nonlinear effect in presence of $\mathbf{N}$-methylephedrine as chiral ligand}

The NME and NBE ligands only differ by the N-methyl group being changed by an N-benzyl group. Therefore, we suspected that NME might generate diastereomeric and aggregationinduced perturbations in the same way as in Figure 1 for the same reaction. The ligand has been previously applied in asymmetric alkylations using $\mathrm{ZnEt}_{2}$ as reagent ${ }^{14-17}$ but, to the best of our knowledge, $\mathrm{ZnMe}_{2}$ has not been investigated so far. Knowing that the NBE ligand displays a more pronounced NLE with $\mathrm{ZnMe}_{2}$ than with $\mathrm{ZnEt}_{2},{ }^{11}$ we suspected that NME might show a NLE in conjunction with $\mathrm{ZnMe}_{2}$, even though it had been shown previously that there is no apparent NLE with $\mathrm{ZnEt}_{2} .{ }^{18}$ Figure $2 \mathrm{a}$ displays the correlation between the enantiomeric excess of the product $\left(e_{\mathrm{P}}\right)$ and the enantiomeric excess of the ligand $\left(\mathrm{ee}_{\mathrm{L}}\right)$ for the asymmetric addition of dimethylzinc to benzaldehyde using chiral (-)-NME (blue dots). Although its (1R,2S)-configuration is identical to the one of (-)-NBE, it surprisingly yielded mainly the $S$-product $\left(\mathrm{P}_{S}\right)$ while (-)-NBE (orange crosses) gave the $R$-product $\left(\mathrm{P}_{\mathrm{R}}\right)$ under similar reaction conditions. The NME-dataset in Figure 2a generates a negative non-linear effect [(-)-NLE], albeit in an apparently inverted manner when compared to common representations of (-)-NLEs (this is due to eep being defined here as the enantiomeric excess of $P_{R}$, which leads to negative ee $P_{P}$ values when $P_{S}$ is predominant).

In parallel, we conducted a screening of the catalyst loading using enantiopure (-)-NME (Figure 2b, blue dots). While a catalytic loading of $20 \mathrm{~mol} \%$ (-)-NME gave the product in $-11 \%$ ee ( $P_{s}$ being predominant), lowering the catalyst loading progressively displaced ee into the positive scale, giving $P_{R}$ in $+18 \%$ ee at $2.5 \mathrm{~mol} \%$. The shape of the curve was found similar to the one observed when using NBE (orange crosses), as well as the eep increase from 20 mol\% to 2.5 mol\% (NME: +31\%; NBE: +33\%). 


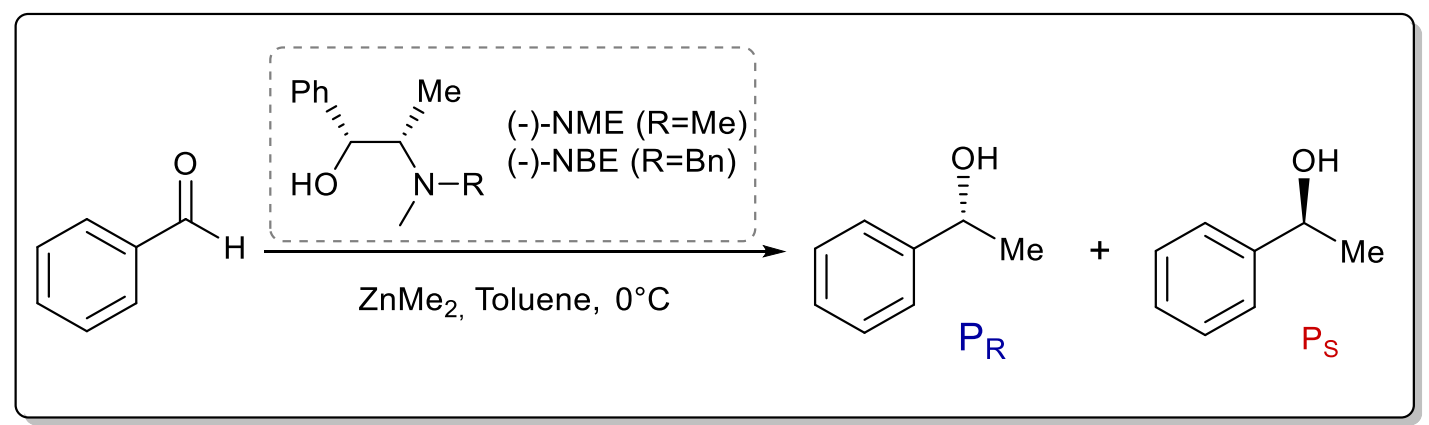

a) Ligand: 20 mol\%, var. ee $\mathrm{ZnMe}_{2}: 1.2$ equivalents b) Ligand: $2.5-20 \mathrm{~mol} \%, 100 \%$ ee $\mathrm{ZnMe}_{2}: 1.025-1.2$ equivalents
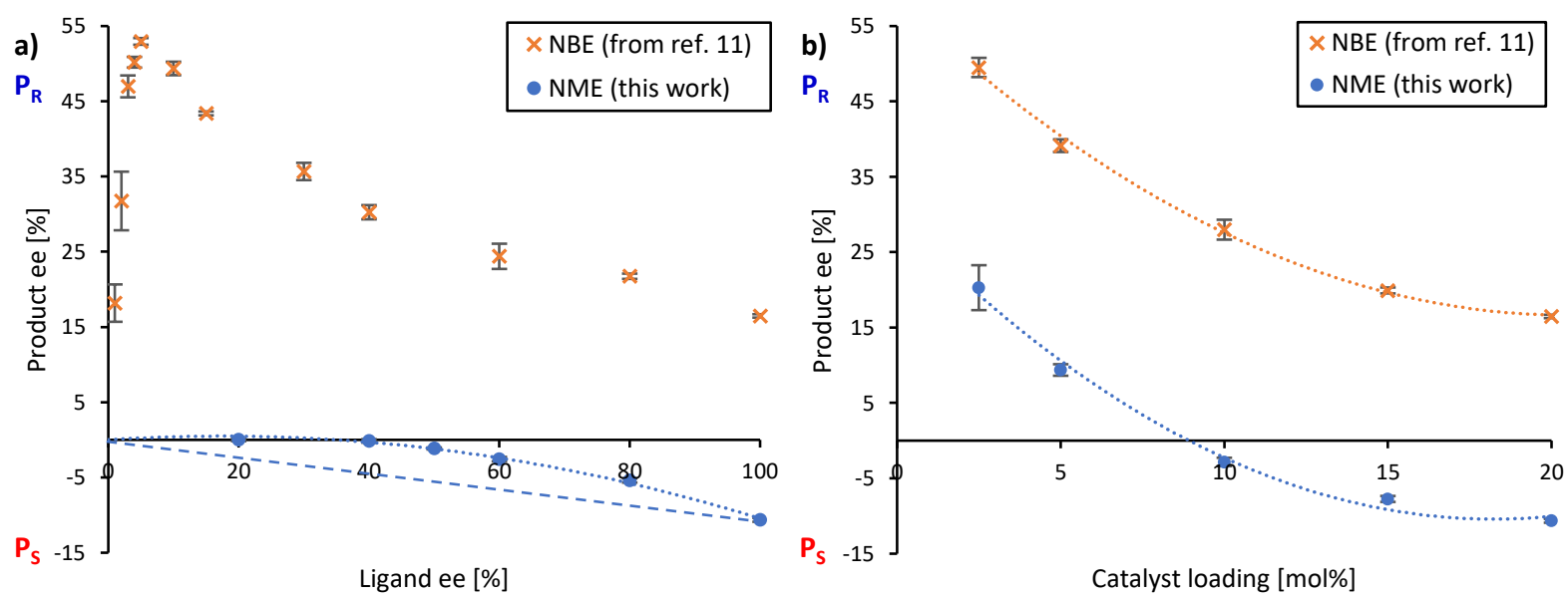

Figure 2. a) NLE-curve and b) eep vs catalyst loading-plot of the (-)-NME- (blue dots) and (-)-NBE- (orange crosses, from ref. 11) catalyzed enantioselective addition of $\mathrm{ZnMe}_{2}$ to benzaldehyde at $0^{\circ} \mathrm{C}$. Each point is the mean of three independent experiments; the vertical bars depict standard deviations. The second-order polynomial fits (dotted lines) serve as visual guidelines. The dashed line in a) simulates a linear eep/ee relationship for the NME-catalysed reaction. The product ee is defined as $\left(P_{R}-P_{S}\right) /\left(P_{R}+P_{s}\right)$.

Such behaviour with enantiopure NME, similar to what observed with the NBE ligand, suggests that both systems follow an analogous catalytic scheme, where both monomeric and dimeric complexes are catalytically active. However, in contrast to NBE the catalytic runs with scalemic NME ligand were homogeneous with no apparent precipitate in the reaction mixture. This may account for the observed differences in NLE curves in Figure 2a. While the hyperpositive NLE with NBE ligand was caused by the precipitation of the RS-dimer (leaving $R$ and RR complexes only in solution and allowing high asymmetric amplification), the (-)-NLE with NME ligand might be the result of the presence of all catalytic species in solution (including S, SS and the meso dimer RS). 
Moreover, the observed negative ee $e_{P}$ at high catalyst loading unambiguously indicates that the dimeric NME-catalyst is not only less enantioselective, it even yields mainly the opposite enantiomeric product than the monomeric catalyst. Catalysis in which both enantiomeric products may be obtained from the same catalyst enantiomer is called enantiodivergent catalysis. It has attracted a lot of attention as a means to access easily both product enantiomers. Over the last two decades, many examples have been reported in which slight changes of the catalyst (substituent, metal, counter-ion, etc.) or of the reaction conditions (solvent, temperature, additive, ligand-to-metal ratio) have inverted the stereochemistry of the product. ${ }^{19-22}$ In our case, the switch which allows to toggle between both product enantiomers is the catalyst concentration, a factor which has not been discussed in this context so far. ${ }^{23}$

These results made us wonder whether it is possible to switch the product enantiomer's

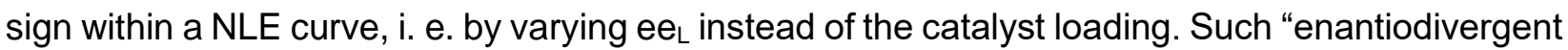
non-linear effect" has already been observed in other catalytic systems ${ }^{24-26}$, but their origin has never been studied; even the possible presence of two catalysts yielding opposite enantiomers has not been proposed. ${ }^{27}$ Therefore, we continued our studies on NME by performing a temperature screening of the catalysis with the enantiopure ligand (Figure 3a, blue dots) or only $50 \%$ ee (orange triangles). At low temperature $\left(0^{\circ} \mathrm{C}\right)$, the product ee of the enantiopure ligand was well below the $e_{p}$ of the scalemic sample, both being negative. Increasing the temperature increased eep in both cases but not in the same manner: the difference between $100 \%$ and $50 \%$ ee $L$ decreased progressively. At $40^{\circ} \mathrm{C}$ the enantiopure ligand even surpassed the $50 \%$ ee $\mathrm{L}^{-}$ sample.

However, an interesting situation occurred at room temperature $\left(20-25^{\circ} \mathrm{C}\right)$ : here, (-)-NME in $50 \%$ ee gave positive eep-values (i.e. $R$ product) while the enantiopure ligand stayed negative (i.e. $S$ product): this is nothing but the requirement for an enantiodivergent non-linear effect. The full NLE curve at room temperature (Figure 3b, blue dots) confirmed this observation: the curve starts at $0 \%$ ee $e_{L}$ in the positive ee $e_{\mathrm{P}}$-range, reaches a maximum, then falls down to cross the ee $\mathrm{L}_{\mathrm{L}}$ axis (at ee $e_{L}$ of ca. $80 \%$ ) and ends up in the negative part of the eep-scale. The use of (+)-NME (red squares) gave the appropriate mirror image of this curve. Thus, going to room temperature changes the (-)-NLE to a hyperpositive NLE, which in addition is also enantiodivergent. 


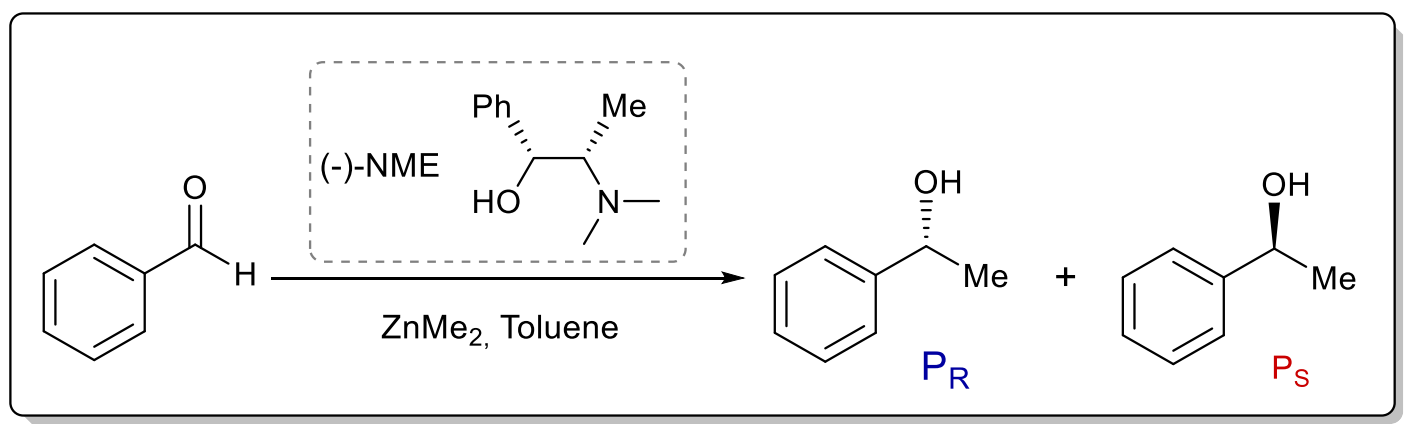

a) (-)-NME: $20 \mathrm{~mol} \%, 100 \%$ or $50 \%$ ee $\mathrm{ZnMe}_{2}: 1.2$ equivalents Var. temperatures

b) (-) or (+)-NME: 20 mol\%, var. ee $\mathrm{ZnMe}_{2}: 1.2$ equivalents Temperature: $20-25^{\circ} \mathrm{C}$
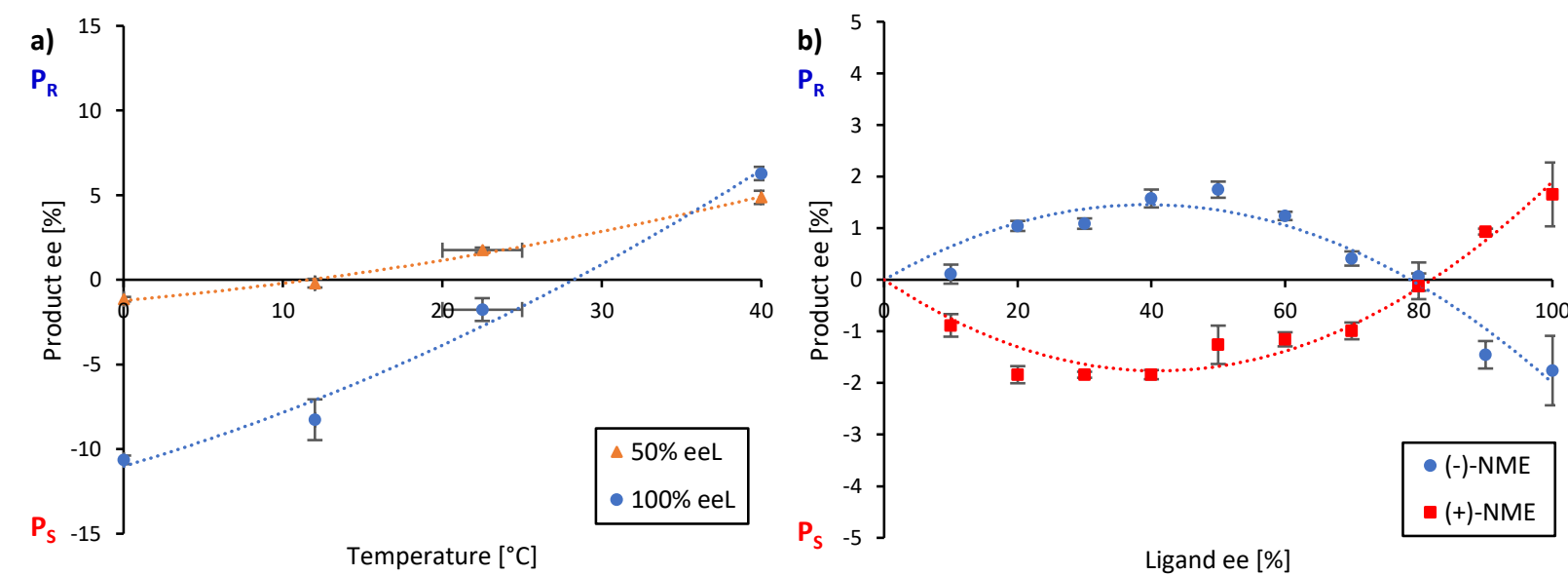

Ligand ee [\%]

Figure 3. a) eep as a function of the reaction temperature (blue dots: $100 \%$ ee $\mathrm{L}_{\text {; }}$ orange triangles: $50 \%$ ee $\mathrm{L}$ ) and b) NLE at room temperature of (-)-NME (blue dots) and (+)-NME (red squares) of the NME-catalysed enantioselective addition of $\mathrm{ZnMe}_{2}$ to benzaldehyde. Each point is the mean of three different experiments; the vertical bars depict standard deviations. The second-order polynomial fits (dotted lines) serve as visual guidelines. The product ee is defined as $\left(\mathrm{P}_{R}-\mathrm{PS}_{S}\right) /\left(\mathrm{P}_{\mathrm{R}}+\mathrm{P}_{S}\right)$.

\section{Model studies: product ee vs catalyst concentration (enantiopure system)}

In the past, non-linear effects have been simulated and quantified using mathematical expressions of the considered models, such as the Kagan $\mathrm{ML}_{n}$ models (catalysis by aggregate) ${ }^{7}$ or the Noyori model (catalysis by monomer, dimer acting as inactive reservoir). ${ }^{9}$ These models have been further extended (electron-rich substrates, ${ }^{28}$ product inhibition ${ }^{29}$ for the Noyori model; monomers as reservoir species with catalytically active homochiral aggregates by Kagan ${ }^{10}$ ). However, to the best of our knowledge there has been no attempt to unify both approaches and 
to consider both monomers and dimers being simultaneously catalytically active ${ }^{30}$ In order to fill this gap and to get a better understanding of hyperpositive and, in particular, enantiodivergent NLEs, we developed mathematical models which allow us to simulate eep $v s$ catalyst concentration- and eep vs eet-plots. We begin with Model I, which is based on an enantiopure system where an enantiopure ligand reacts with a metal to give monomeric and dimeric homochiral complexes ( $R$ and $R R$, respectively) which both catalyse the reaction at different rates $\left(k_{1}\right.$ and $\left.k_{2}\right)$ and with different enantioselectivities ( $e_{1}$ and $\left.e e_{2}\right)$, as shown in Figure 4; [Cattot] represents the total catalyst concentration. For the sake of simplicity, we assume that both $\mathrm{R}$ and RR-catalysts follow a similar mechanism with a rate law of type $-d[S u b] / d t=k_{i}[$ Cat $]$ [Sub][Rea] (with $\mathrm{k}_{\mathrm{i}}$ and [Cati] being the respective rate constants and catalyst concentrations, [Sub] and [Rea] the substrate and reactant concentrations; all species are first-order). We also assume that the $[R R] /[R]-$ ratio stays constant over the course of the reaction and depends only on the homochiral dimerization constant $\mathrm{K}_{\text {Homo. }}{ }^{31}$ This makes Model I reminiscent of Kagan's $\mathrm{ML}_{n}$ model, which also considers the ratio between different catalytic species to be constant over time. The case of a time-dependent $[R R] /[R]-$ ratio will be discussed at the end of this study.

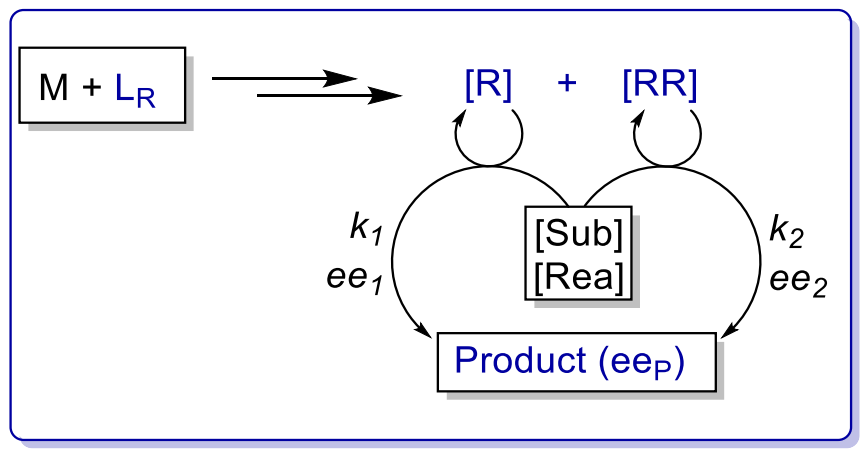

$$
\begin{gathered}
\mathrm{R}+\mathrm{R} \stackrel{\mathrm{K}}{\stackrel{\mathrm{K}_{\text {Homo }}}{\rightleftharpoons}} \mathrm{RR} \\
\mathrm{K}_{\text {Homo }}=\frac{[\mathrm{RR}]}{[\mathrm{R}]^{2}} \\
{\left[\mathrm{Cat}_{\text {tot }}\right]=[\mathrm{R}]+2[\mathrm{RR}]}
\end{gathered}
$$

Figure 4. Schematic representation of Model I, which consists of a monomeric $(R)$ and a dimeric (RR) enantiopure catalyst that both operate at a steady state and are linked through the equilibrium constant $K_{\text {Homo. }}$. The catalysts are issued from the reaction of a metal salt $(M)$ with a chiral, enantiopure ligand $\left(L_{R}\right)$ and promote the reaction of a substrate (Sub) and a reactant (Rea) to form a chiral product with the overall enantiomeric excess eep. The R- and RR-catalysts yield a product with a rate constant of $\mathrm{k}_{1}$ and $\mathrm{k}_{2}$, respectively, and with an enantioselectivity of ee 1 and ee 2. 


$$
\begin{gathered}
e e_{P}=\frac{e e_{1}+\gamma \frac{k_{2}}{k_{1}} e e_{2}}{1+\gamma \frac{k_{2}}{k_{1}}} \\
\gamma=\frac{\sqrt{1+8 K_{\text {Homo }}\left[C a t_{t o t}\right]}-1}{4}
\end{gathered}
$$

By combining the set of equations displayed in Figure 4, it was possible to obtain equations (1) and (2) which relate ee $e_{p}$ to the parameters $k_{2} / k_{1}, e e_{1}, e e_{2}, K_{\text {Homo }}$ and [Cattot], and allowed us to compute ee vs [Cattot-curves. Figure 5a displays the evolution of ee $\mathrm{p}_{\mathrm{p}}$ for selected values of $\mathrm{ee}_{2}$ with fixed values of $\mathrm{K}_{\text {Homo }}, \mathrm{k}_{1}$ and $\mathrm{k}_{2}\left(\mathrm{k}_{2} / \mathrm{k}_{1}\right.$ was set to 1 , ee $\mathrm{e}_{1}$ to $100 \%$ and $\mathrm{K}_{\text {Homo }}$ to 33 , which corresponds to the association constant of DAIB-ZnMe).,32 The graphs show that an enantiodivergent behaviour can indeed be observed by varying [Cattot], as long as $\mathrm{ee}_{2}$ has a sufficiently low and negative value. This is further favoured by high values for $\mathrm{K}_{\text {Homo }}$ (Figure $5 \mathrm{~b}$ ) and $k_{2} / k_{1}$ (Figure $5 c$ ): the more the dimeric complex prevails over the monomer and the higher its relative activity, the more the curve becomes hyperbolic, pushing the point at which it crosses the [Cat ${ }_{\text {tot }}$-axis (i.e. ee $\mathrm{P}_{\mathrm{P}}=0$, labelled as $\left[\mathrm{Cat}_{\text {tot }}\right]^{0}$ ) to lower [Cat ${ }_{\text {tot }}$. [Cat ${ }_{\text {tot }}{ }^{0}$ corresponds to an overall catalytic system where $\mathrm{R}$ and $\mathrm{RR}$ catalysts compensate each other to yield an overall racemic product - even if both give independently enantiopure products. 

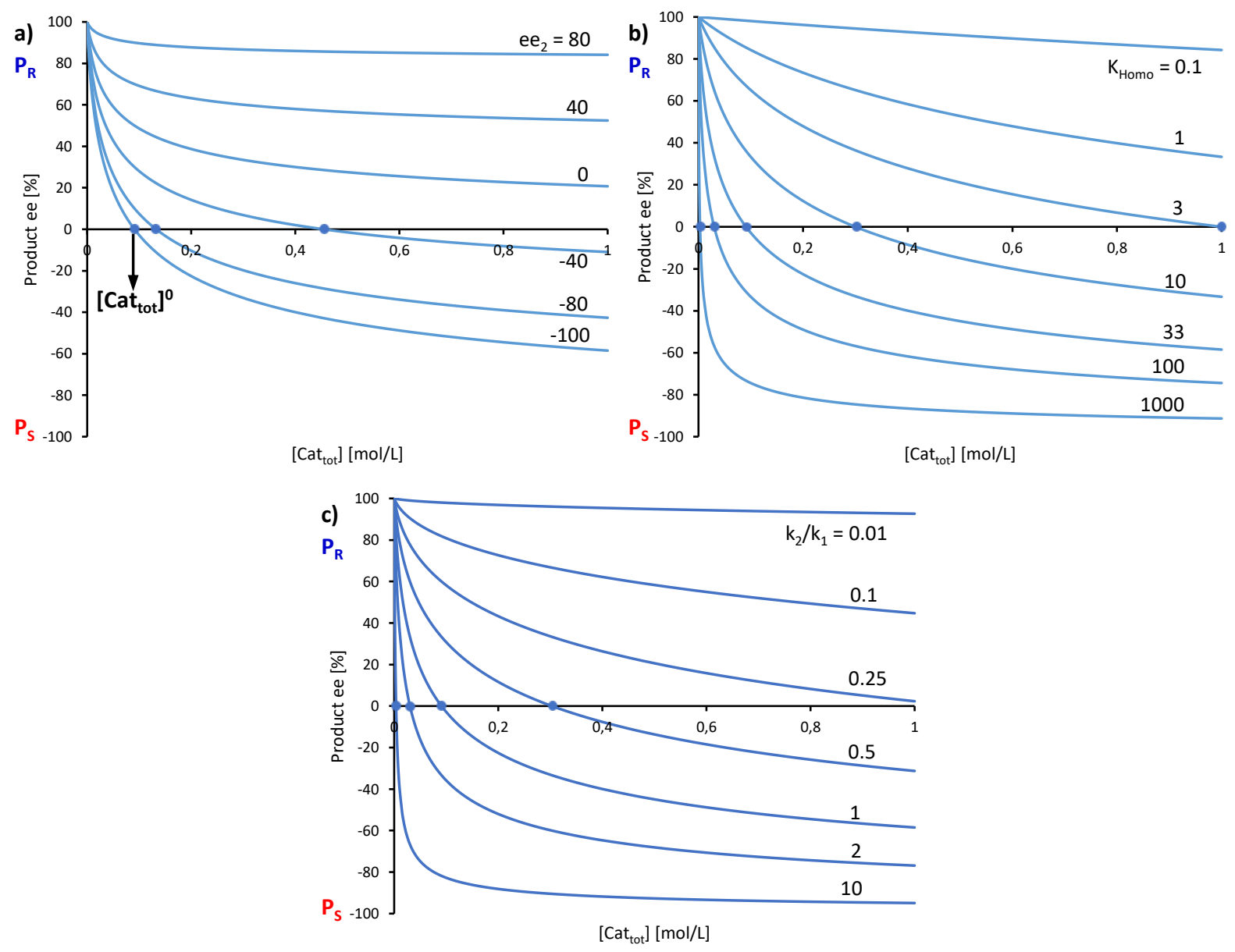

Figure 5. Simulation of the relationship between eep and [Cattot] according to equations (1) and (2). The basic set of parameters is ee $1=100, e_{2}=-100, K_{\text {Homo }}=33$ and $k_{2} / k_{1}=1$. Each panel shows curves where one of the parameters has been varied: a) ee $\left.2, b) K_{\text {Homo }}, c\right) k_{2} / k_{1}$. The product ee is defined as $\left(P_{R^{-}}\right.$ $P S) /\left(P_{R}+P_{S}\right)$. 


\section{Model studies: product ee vs ligand ee (scalemic system)}

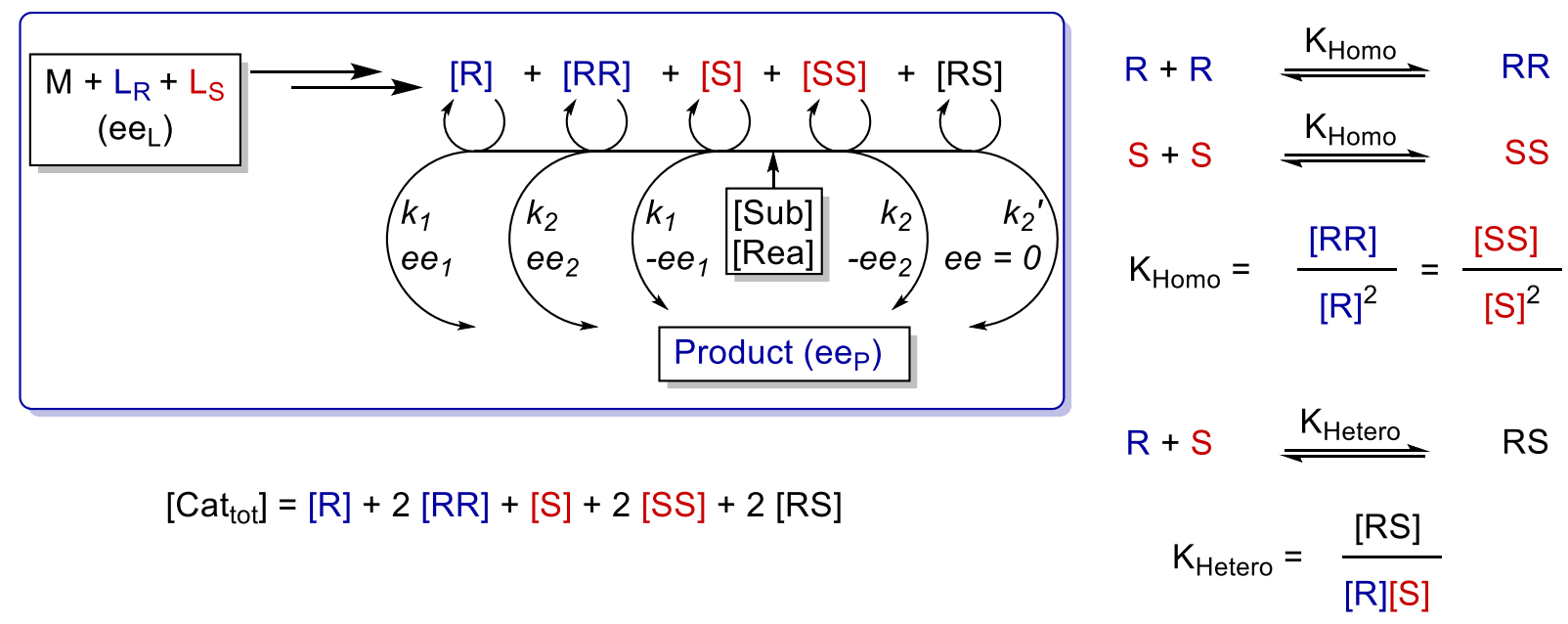

Figure 6. Schematic representation of Model II, which consists of monomeric $(R, S)$ and both homo- (RR, SS) and heterochiral (RS) dimeric catalysts that all operate at a steady state and are linked through the equilibrium constants $\mathrm{K}_{\text {Homo }}$ and $\mathrm{K}_{\text {Hetero. }}$. The catalysts are issued from the reaction of a metal salt (M) with a mixture of both ligand enantiomers ( $L_{R}$ and $\left.L_{S}\right)$ with an enantiomeric excess of eet. They promote the reaction of a substrate (Sub) and a reactant (Rea) to form a chiral product with the overall enantiomeric excess eep. The monomeric, the homochiral dimeric and heterochiral dimeric catalysts yield a product with a rate constant of $k_{1}, k_{2}$, and k2', respectively, and with an enantioselectivity of ee $e_{1}$ and ee $(R$ and $R R)$ or $\mathrm{ee}_{1}$ and $-\mathrm{ee}_{2}(\mathrm{~S}$ and $\mathrm{SS})$. The RS dimer yields racemic product $(\mathrm{ee}=0)$.

In order to simulate also NLE curves, we expanded Model I to non-enantiopure ligands, as shown in Figure 6. The resulting system (Model II) now includes the catalytic species S and SS (which, like their enantiomeric counterparts, are linked through $\mathrm{K}_{\text {Homo }}$ and catalyse with the kinetic constants $k_{1}$ and $k_{2}$ ) and also the heterochiral dimer RS, which is related to $R$ and $S$ through the dimerization constant $\mathrm{K}_{\text {Hetero }}$ and may generate racemic products with a rate constant $\mathrm{k}_{2}$. Thus, a single scalemic ligand gives rise to 5 different catalytic species. To derive closed mathematical expressions, we followed the approach used by Noyori for the DAIB-model which consists in introducing $\alpha=[R]+[S]$ and $\beta=[R][S]$ to simplify the equations. ee $e_{P}$ and $e_{L}$ are then given by equations (3) and (4) as functions of $\alpha$ and $\beta$. Since $\beta$ is itself a function of $\alpha$ [cf. equation (5)], ee and $e_{L}$ are linked through $\alpha$ and depend only on the parameters $K_{H_{0 m o}}, K_{H e t e r o}, k_{1}, k_{2}, k_{2}$, ee $e_{1}, e_{2}$ and [Cat ${ }_{\text {tot }}$. After defining these parameters, ee $e_{P} v s e_{L}$-datasets could be obtained by choosing appropriate values for $\alpha$ (cf. Supplementary Methods for the details of the calculations and the general expressions for the upper and lower limits of $\alpha$ ). For this study, we will focus on the cases where the NLE is hyperpositive and potentially enantiodivergent - that is with $\mathrm{K}_{\text {Hetero }}>2 \mathrm{~K}_{\text {Homo }}$ which 
is, as in the Noyori model, a necessary condition to obtain (+)-NLEs - and $\mathrm{ee}_{1}>\mathrm{ee}_{2 .}{ }^{9}$ Figure 7 shows several cases computed from Model II. To simplify the discussion we have set $k_{2}{ }^{\prime}=0$ in all simulations except in Figure $7 f$.

$$
\begin{gathered}
e e_{P}=\frac{\sqrt{\alpha^{2}-4 \beta}\left(e e_{1}+\alpha K_{\text {Homo }} \frac{k_{2}}{k_{1}} e e_{2}\right)}{\alpha+\left(\alpha^{2}-2 \beta\right) K_{\text {Homo }} \frac{k_{2}}{k_{1}}+\beta K_{\text {Hetero }} \frac{k_{2}{ }^{\prime}}{k_{1}}} \\
e e_{L}=\frac{\sqrt{\alpha^{2}-4 \beta}\left(1+2 \alpha K_{\text {Homo }}\right)}{\left[\text { Cat }_{\text {tot }}\right]} \\
\beta=\frac{\left(\alpha+2 K_{\text {Homo }} \alpha^{2}-\left[\text { Cat }_{\text {tot }}\right]\right)}{4 K_{\text {Homo }}-2 K_{\text {Hetero }}}
\end{gathered}
$$

Influence of $\mathbf{K}_{\mathrm{Homo}}$, [Cat ${ }_{\text {tot }}$ ], $\mathbf{k}_{\mathbf{2}} / \mathbf{k}_{\mathbf{1}}$ and $\mathbf{e} \mathbf{e}_{\mathbf{1 / 2}}$. A non-linear effect is hyperpositive as long as the highest product ee (labelled as $e_{\mathrm{P}}{ }^{\max }$ ) is different from the ee $e_{\mathrm{P}}$ for the enantiopure ligand $\left(\mathrm{ee}_{\mathrm{P}}{ }^{100}\right.$ ). In Model II, ee ${ }^{100}$ will be strongly dependent on $\mathrm{K}_{\text {Homo, }}$ [Cat ${ }_{\text {tot }}$ ] and $\mathrm{k}_{2} / \mathrm{k}_{1}$ (Figure $7 \mathrm{a}-\mathrm{c}$ ): the higher $\mathrm{K}_{\text {Homo, }}$ [Cat ${ }_{\text {tot }}$ or $\mathrm{k}_{2} / \mathrm{k}_{1}$, the lower $e_{\mathrm{P}}{ }^{100}$. This is consistent with a higher proportion and a higher

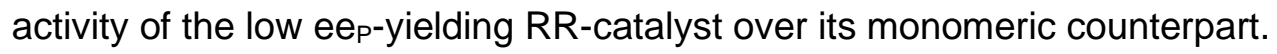

In all representations in Figure 7, we selected conditions in which $e^{100}$ could be negative and where the NLE curve crosses the ee $e^{e}{ }_{P}^{100}$, the lower the crossing point at which $e_{P}=0\left(e_{L}{ }^{0}\right)$. At this point, the outcomes of all catalysts compensate each other to yield a racemic product. $e_{P}{ }^{m a x}$ diminishes as $e_{P}{ }^{100}$ decreases; the maximum's ee $e_{L}-v_{a l u e}\left(e_{L}{ }^{m a x}\right)$ is only slightly affected by $K_{\text {Homo }}$ and, to a somewhat greater extend, by [Cat tot] and $k_{2} / k_{1}$. This is seen nicely if, for a given set of parameters, $K_{\text {Homo }}$ is

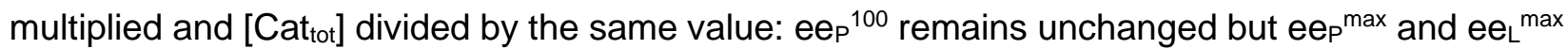
do not (cf. Supplementary Figure 1). Figure $7 d$ shows the impact of $e e_{1}$ and $e e_{2}$ on $e_{P}{ }^{100}$ : the lower $e_{2}$, the lower $e e^{100}$. This is also true for $e e_{1}$; however, if both $e_{1}$ and $e_{2}$ are negative, the enantiodivergent NLE curve becomes a classic (+)-NLE for the S-product $\left(P_{S}\right.$, Figure $7 d$, dashed curves); the same holds for the R-product $\left(P_{R}\right)$ if $e_{1} \leq e_{2}$ and if both are positive. In a similar way, very high $\mathrm{K}_{\mathrm{Homo}^{-}}$, [Cat ${ }_{\text {tot }}$ - or $\mathrm{k}_{2} / \mathrm{k}_{1}$-values lead to apparent $(+)-\mathrm{NLES}$ as ee ${ }^{\max }$ and ee $^{\text {max }}$ 
become exceedingly close to 0 . Lowering the absolute amount of both a positive $\mathrm{ee}_{1}$ and a negative $e_{2}$ leads to a compression of the spectra (cf. Supplementary Figure 2).

Influence of $\mathbf{K}_{\text {Hetero }}$ and $\mathbf{k}_{\mathbf{2}}$ '. In contrast to the previously discussed parameters, an increase in

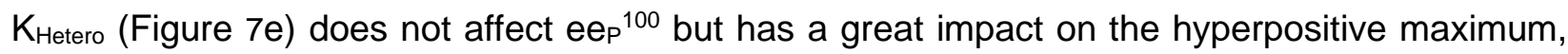
which is shifted to higher $e_{\mathrm{P}}{ }^{\max }$ and lower $e_{\mathrm{L}}{ }^{\max }$ values. Consequently, the $e_{\mathrm{L}}{ }^{0}$ is shifted to higher $e_{L}$ values under the same conditions. However, this is only true if the meso dimer is catalytically inactive: RS performing racemic catalysis $\left(k_{2}{ }^{\prime} / k_{1} \neq 0\right.$, Figure $\left.6 f\right)$ leads to the inverse effect, namely a compacting of the curve. The values of $e_{P}{ }^{100}$ and $e^{0}{ }^{0}$ remain unchanged, the latter being an isobestic point. The value of $e{ }^{\max }$ decreases significantly even at low $\mathrm{k}_{2}{ }^{\prime} / \mathrm{k}_{1}$ as the concentration of $\mathrm{RS}$ at low ee $\mathrm{L}$ is particularly high. $\mathrm{k}_{2}^{\prime} / \mathrm{k}_{1}$-values higher than $\mathrm{k}_{2} / \mathrm{k}_{1}\left(\mathrm{k}_{2}{ }^{\prime} / \mathrm{k}_{1}>1\right.$ in Figure $\left.7 \mathrm{f}\right)$ additionally lead to a contraction of the curve between $e^{\circ}{ }^{0}$ and $e e_{L}=100$ and push its appearance

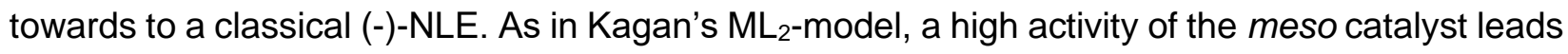
to asymmetric depletion. 

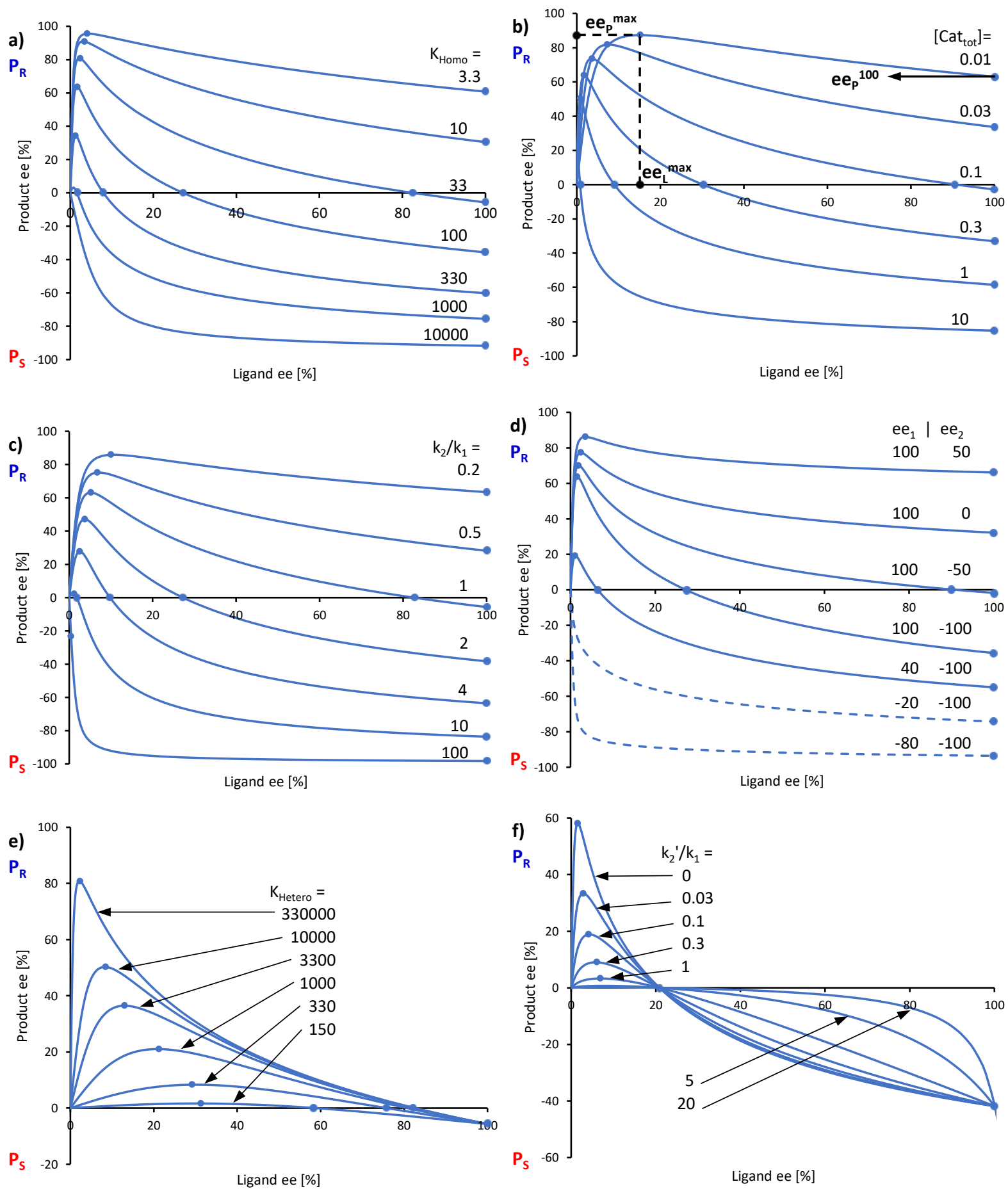

Figure 7. Simulation of NLEs with Model II, varying parameters a) $K_{\text {Homo, }} b$ ) [Cattot], c) $k_{2} / k_{1}$, d) ee end $_{1}$ and e) $K_{\text {Hetero }}$ and f) $k_{2}{ }^{\prime} / k_{1}$. Fixed parameters: [Cattot] $=0.11, k_{2} / k_{1}=1, e e_{1}=100, e e_{2}=-100$ and $k_{2}{ }^{\prime} / k_{1}=0$ in all curves except where the corresponding parameter is varied; $K_{\text {Homo }}=33$ (b, c, e), 100 (d) and 130 (f); $K_{\text {Hetero }}$ $=330000(a, d, f), 100000$ (b) and 33000 (c). The product ee is defined as $\left(P_{R}-P_{S}\right) /\left(P_{R}+P_{S}\right)$. 


\section{Dynamic properties of Models I and II}

Apart from simulating ee $e_{P}$ vs ee kinetic properties of the systems. This can be useful as some kinetic features may be characteristic for one or the other model, and thus be used as an additional probe to support the validity of a model for a specific catalytic reaction. Blackmond showed this in conjunction with the Kagan and Noyori models; ${ }^{33,34}$ Micheau and co-workers even built a toolset, using the different kinetic properties of both systems, which allows an easy distinction between monomer- and dimercatalysed enantioselective systems (which is not necessarily possible on the basis of ee $v_{\mathrm{P}}$ ee $_{\mathrm{L}}$ curves alone $)^{35}$ and verified the origin of the non-linearity in the Noyori model by a kinetic system based on differential equations. ${ }^{36}$ This prompted us to extend our work on Models I and II to a kinetic study.

The mathematical expressions of the rate laws based on Models I and II turned out to correspond to a standard second-order rate law (equations (6) and (7)), albeit with a more complex term for kobs (cf. Supplementary Methods for the calculation details). For aggregate-free catalysed reactions, with $1^{\text {st }}$ order in catalyst, $\mathrm{k}_{\mathrm{obs}}$ is the product of [Cat ${ }_{\text {tot }}$ ] and the rate constant $\mathrm{k}_{1}$ (equation (8)). According to Model I, where monomer and dimer coexist and which are both catalytically active, $\mathrm{k}_{\mathrm{obs}}$ depends on the parameters previously discussed: the (now absolute instead of relative) rate constants $k_{1}$ and $k_{2}, K_{\text {Homo }}$ and [Cat ${ }_{\text {tot }}$ (equation (9)). This holds for chiral, enantiopure catalysts as well as for achiral ones, since we consider only the system's kinetic behaviour and not a possible product ee. Non-enantiopure catalytic systems following Model II additionally depend on ee $\mathrm{L}_{\mathrm{L}} \mathrm{k}_{2}$ ' and $\mathrm{K}_{\text {Hetero }}$ (equation (10)). For the sake of simplicity, we will consider here only the second-order rate law where the substrate and reactant concentrations are equal ([Sub] = [Rea]), however the following discussion applies also to its more general form where both differ from each other.

General second-order rate law (if $[S u b]=[R e a]$ ): $-\frac{\mathrm{d}[\mathrm{Sub}]}{\mathrm{dt}}=\mathrm{k}_{\mathrm{obs}}[\mathrm{Sub}]^{2}$

Integrated form of the second-order rate law: $[\mathrm{Sub}]_{\mathrm{t}}=\left(\frac{1}{[\mathrm{Sub}]_{0}}+\mathrm{k}_{\mathrm{obs}} \mathrm{t}\right)^{-1}$

If only monomers exist: $\mathrm{k}_{\mathrm{obs}}=\mathrm{k}_{1}\left[\mathrm{Cat}_{\mathrm{tot}}\right]$ 
If monomers and dimers catalyse (Model l, achiral or enantiopure catalyst):

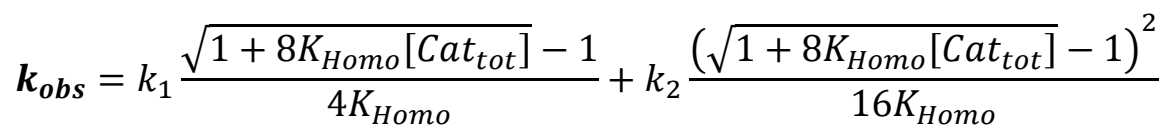

If monomers and dimers catalyse (Model II, chiral catalyst with any $e_{L}$ ):

$$
\begin{aligned}
& \boldsymbol{k}_{\text {obs }}=k_{1} \alpha+k_{2} K_{\text {Homo }}\left(\alpha^{2}-2 \beta\right)+k_{2}^{\prime} K_{\text {Hetero }} \beta \\
& \text { With ee }_{L}=\frac{\sqrt{\alpha^{2}-4 \beta}\left(1+2 \alpha K_{\text {Homo }}\right)}{\left[\text { Cat }_{\text {tot }}\right]} \text { and } \beta=\frac{\left(\alpha+2 K_{\text {Homo }} \alpha^{2}-\left[\text { Cat }_{\text {tot }}\right]\right)}{4 K_{\text {Homo }}-2 K_{\text {Hetero }}}
\end{aligned}
$$

The interesting point here is that [Cat $\left.{ }_{t o t}\right]$ and $e_{\llcorner}$are user-controlled parameters, whose variation leads to singular changes in $\mathrm{k}_{\text {obs. }}$. This can be seen in simulated $\mathrm{k}_{\text {obs }} /\left[\mathrm{Cat}_{\text {tot }}\right.$ ] vs [Cat ${ }_{\text {tot }}$ plots: in the case of aggregate-free catalysed reactions (cf. equation (8)) such a plot results in a flat line, whose $y$-intercept is equal to $k_{1}$ (Figure $8 a$, blue line). The same is observed for Model I (equation 9 ) in a special case that is when $k_{2} / k_{1}=2$ : the loss of monomeric catalyst upon increase of [Cat ${ }_{\text {tot }}$, because of dimeric aggregation, is then perfectly compensated by the higher activity of the dimer catalyst. Otherwise, an increase in [Cattot] leads to a significant change of $\mathrm{k}_{\mathrm{obs}} /\left[\mathrm{Cat} \mathrm{t}_{\mathrm{tot}}\right]$ : it increases if $k_{2} / k_{1}>2$ (grey dashed line) or decreases if $k_{2} / k_{1}<2$ (orange dotted line), which is symptomatic of the changing $[R R] /[R]$-ratio. At very low [Cat tot] the amount of RR-catalyst becomes negligible and $\mathrm{k}_{\mathrm{obs}} /\left[\mathrm{Cat}_{\text {tot }}\right]$ becomes equal to $\mathrm{k}_{1}$; on the other hand, $\mathrm{k}_{\mathrm{obs}} /\left[\mathrm{Cat} \mathrm{t}_{\text {tot }}\right]=0.5 \mathrm{k}_{2}$ at very high [Cat ${ }_{\text {tot }}$ because of the prevalence of the dimeric catalyst. Thus, $\mathrm{k}_{\mathrm{obs}} /\left[\mathrm{Cat} \mathrm{t}_{\text {tot }}\right]$ varies over changing [Cattot] even if $R$ and $R R$ catalyse with the same rate $\left(k_{1}=k_{2}\right.$, green dashed/dotted line). With nonenantiopure ligands $\mathrm{k}_{\text {obs }} /\left[\mathrm{Cat}_{\text {tot }}\right]$ varies in a similar way as in Figure $7 \mathrm{a}$ and depends, in addition, also on k2' (cf. Supporting Figure 3 for a commented example with $e_{L}=0$ ).

The other user-controlled parameter, ee $\mathrm{L}_{\mathrm{L}}$ also influences $\mathrm{k}_{\mathrm{obs}}$ (at constant [Cat $\mathrm{t}_{\text {tot }}$ ) as seen in $\mathrm{k}_{\mathrm{obs}}$ vs ee $\mathrm{e}$ plots (Figure $8 \mathrm{~b}$, computed from equation (10)). $\mathrm{k}_{\mathrm{obs}}$ is constant if $\mathrm{k}_{2} / \mathrm{k}_{1}=\mathrm{k}_{2}{ }^{\prime} / \mathrm{k}_{1}=2$ (blue full line). The case of $k_{2} / k_{1}$-values higher than 2 leads to an increase $k_{o b s}$ especially at high $\mathrm{ee}_{\mathrm{L}}$, where the concentration of the homochiral dimers is also higher, and results in a positive slope (orange dotted line). On the other hand, increasing $k_{2}{ }^{\prime} / k_{1}$ gives a negative slope as it affects $\mathrm{k}_{\mathrm{obs}}$ mostly at low ee $\mathrm{L}$, where the proportion of RS-dimers is highest (grey dashed line). A simultaneous increase of $\mathrm{k}_{2}{ }^{\prime} / \mathrm{k}_{1}$ and $\mathrm{k}_{2} / \mathrm{k}_{1}$ by the same amount also yields a negative slope if $\mathrm{K}_{\text {Hetero }}$ $>2 \mathrm{~K}_{\text {Homo }}$ (green dashed/dotted line). If the $\mathrm{K}_{\text {Hetero }} / \mathrm{K}_{\text {Homo-relationship is inverted then a positive }}$ slope is obtained, cf. Supplementary Figure 4. 

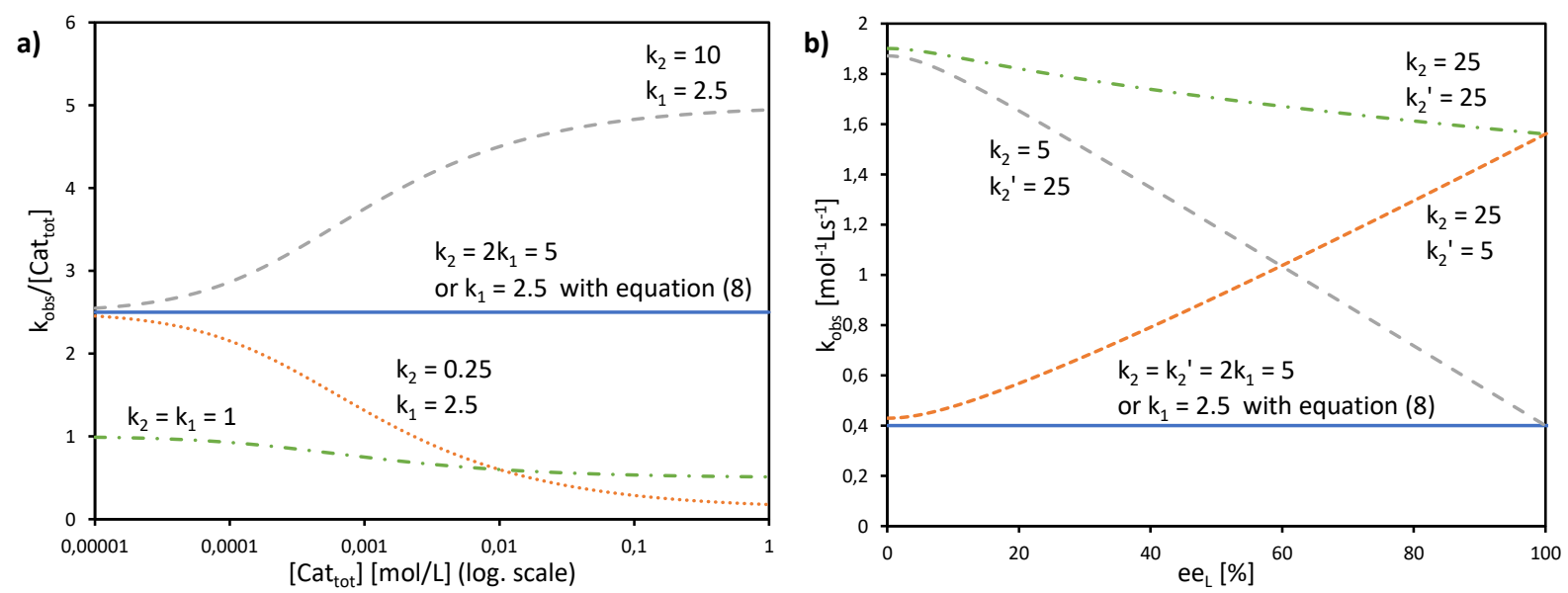

Figure 8. a) Simulated $\mathrm{k}_{\text {obs }} /\left[\mathrm{Cat}\right.$ tot] $v s$ [Cattot]-plots computed from equation (9) (KHomo $=1000, \mathrm{k}_{1}$ and $\mathrm{k}_{2}$ as indicated) and b) simulated $\mathrm{K}_{\text {obs }} v s$ ee $\mathrm{L}$ plots computed from equations (10), (4) and (5) ( $\mathrm{K}_{\text {Homo }}=30, \mathrm{~K}_{\text {Hetero }}$ $=3000$, [Cattot $=0.16, k_{1}=2.5, k_{2}$ and $k_{2}^{\prime}$ as indicated). The full blue lines in $a$ ) and $b$ ) can also be obtained from equation (8) (i. e. no aggregates are present in the system, the monomer is the only catalyst) using the indicated $k_{1}$ value.

In our previous study, we also determined the catalyst order $c$ of the NBE-catalysed reaction when considering the system to follow the rate law $-\mathrm{d}[\mathrm{Sub}] / \mathrm{dt}=\mathrm{k}[\mathrm{Cat}$ tot $]$ [Sub][Rea]. ${ }^{11}$ For this, we had determined the catalyst order $c$ using Variable Time-Normalised Analysis (VTNA) ${ }^{37-}$ 40 of rate profiles obtained from enantiopure NBE at different catalyst loadings. Plotting catalyst order $c$ vs catalyst loading gave an unusual $U$-shaped plot which we postulated to originate in a [Cat ${ }_{\text {tot }}$-induced change of $k_{\text {obs. }}$. The present kinetic model now allows us to verify this assumption,

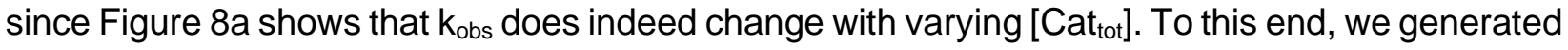
sets of rate profiles from equations (7) and (9) by varying [Cat tot] and leaving all other parameters unchanged. Then, $c$ was determined from two different rate profiles at a time using VTNA; the results are shown in Figure 9. In concordance with the previous discussion, $k_{2} / k_{1}=2$ gives a constant $c$ value while varying the catalyst loading, since $\mathrm{k}_{\mathrm{obs}}$ is constant in that special case. $\mathrm{k}_{2} / \mathrm{k}_{1}$ $<2$ gives a U-shaped curve, here with a minimum at $c \approx 0.7$ (blue dots) and similar to the experimental one obtained from NBE. On the other hand $k_{2} / k_{1}>2$ gives a bell-shaped curve with a maximum at $c \approx 1.5$ (grey triangles). Increasing $\mathrm{K}_{\text {Homo }}$ displaces the curve's extremum to lower catalyst loading values (green crosses). 


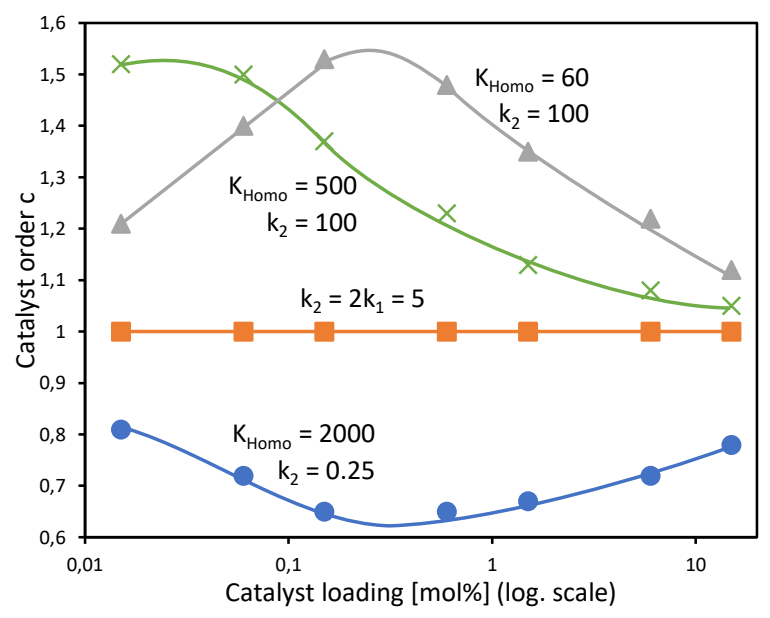

Figure 9. Simulated $c$ vs catalyst loading plot of a catalytic system following the kinetics of Model I and treated as if following the rate law $-\mathrm{d}[\mathrm{Sub}] / \mathrm{dt}=\mathrm{k}\left[\right.$ Cattot $^{\mathrm{c}}[\mathrm{Sub}]^{\mathrm{a}}[\text { Rea }]^{\mathrm{b}}$, with $c$ as a variable partial order in catalyst, with undetermined rate constant $k$ and partial substrate/reactant order $a$ and $b$. Each datapoint relates the $c$-value obtained from two Model I-rate profiles via VTNA (from equations (7) and (9), with [Sub]o $=0.833, \mathrm{k}_{1}=2.5, \mathrm{~K}_{\text {Homo }}$ and $\mathrm{k}_{2}$ as indicated, var. [Cattot]) with the mean of their catalyst loading values; the procedure is described in the Supplementary Methods. The full lines are free-hand drawings which serve as visual guidelines.

\section{Discussion}

Models I and II, although kept conceptually simple, allow to gain a better understanding of several aspects of the NME- and NBE-catalysed reactions, which we will discuss in this section. Globally, the shape of the experimental curves (eep vs catalyst loading as well as eep vs eeL, Figures 2 and 3) corresponds well with the computed curves in Figures 5 and 7. Previously published graphs from reactions with NBE and $\mathrm{ZnEt}_{2}$ as the dialkylzinc reagent, ${ }^{12}$ giving a much weaker hyperpositive NLE than $\mathrm{ZnMe}_{2}$, also concord with the simulated ones in this study. The simulations have also given insights into the differences between the NME- and NBE-based catalysts: the overall lower ee $e_{\mathrm{P}}$-values with NME may be due to lower $\mathrm{ee}_{1}$ or $\mathrm{ee}_{2}$ values, as well as to higher $\mathrm{K}_{\text {Homo }}$ and/or $\mathrm{k}_{2} / \mathrm{k}_{1}$. The slight hyperpositive (and enantiodivergent) NLE with NME at room temperature (Figure $3 b$ ) may arise from $\mathrm{K}_{\text {Hetero }}$ being only slightly higher than $2 \mathrm{~K}_{\text {Homo }}$, since pronounced hyperpositive NLEs were simulated only when $\mathrm{K}_{\text {Hetero }}$ and $\mathrm{K}_{\text {Homo }}$ are highly different (cf. Figure 7e). At $0{ }^{\circ} \mathrm{C}$ (Figure 2a), NME even generates a (-)-NLE which precludes that upon cooling, $\mathrm{K}_{\text {Hetero }}$ falls below the value of $2 \mathrm{~K}_{\text {Homo }}$. 
In the case of NBE (Figure 2a, orange crosses), the strong hyperpositive curve arises from the precipitation of the heterochiral dimer, which has an effect similar to an increase of $\mathrm{K}_{\text {Hetero. }}$. The simulations in Figure 7 reveal also that, even if the dimeric catalyst gives the opposite product enantiomer in its pure form ( $\left(e_{2}=-100 \%\right.$ ), the NLE does not need to be enantiodivergent as long as the dimer's participation in the catalytic process is sufficiently low (due to low $\mathrm{K}_{\text {Homo, }}$, [Cattot] or $k_{2} / k_{1}$, as seen in Figures $\left.7 a-d\right)$. Therefore, dimeric NBE-ZnMe might yield the opposite enantiomer as well, even though its NLE and eep vs catalyst loading-plot (Figure 2, orange crosses) don't show direct evidence for that. This possibility is particularly interesting as it raises questions whether enantiodivergence between monomeric and dimeric catalysts might be a systematic phenomenon. There are various cases of 2:1 ligand-to-metal complexes which have been reported to yield the other product enantiomer than their 1:1 counterparts, ${ }^{41-49}$ Seebach's TADDOL-Ti-complexes being the first (but largely unnoticed) ones. ${ }^{42-44} \quad 2: 1$ complexes can be considered as analogues of dimeric complexes, both are even equivalent in Kagan's $\mathrm{ML}_{2}$ model. ${ }^{7}$

Also, the models suggest that it is possible to obtain racemic products when working at a certain catalyst concentration ([Cattot $]^{0}$, cf. Figure 5) or ligand ee (ee ${ }^{0}$, cf. Figure 7), although the system consists of only highly enantioselective catalytic species. This is well exemplified with the results from NME-catalysed reactions at room temperature (Figure $3 b$ ): the only $2 \%$ ee $e_{P}$ yielded by the enantiopure catalyst may let one think that NME is a totally non-enantioselective catalyst in this reaction, which is definitely not the case.

Furthermore, the kinetic models show that the evolution in the ratios between the different catalytic species, when changing reaction parameters like [Cat ${ }_{t_{0 t}}$ or $e_{\mathrm{L}}$, results in a variation of the rate constant $\mathrm{k}_{\text {obs. }}$. This allowed us to confirm our previous assumption that a U-shaped curve for the catalyst order $c$ vs. catalyst loading plot is consistent with Model I, assuming the monomer has more than half the catalytic activity of the dimer $\left(k_{2} / k_{1}<2\right)$. However, this feature also shows the limitations of Model I and II: the simulated U-shaped $c$ vs catalyst loading-curve (Figure 9) spans over several orders of magnitude of catalyst loading, whereas the experimental one obtained from NBE spans only from 20 to $2.5 \mathrm{~mol} \%{ }^{11}$ For second order kinetic laws, plotting $1 /[\text { Sub }]_{t}$ vs time gives a linear graph, however the corresponding experimental plots from NBEand NME-catalysed reactions are mostly curved (cf. Supplementary Figure 5). This shows that $k_{\text {obs }}$ is not constant over time, most probably because of a change of the catalysts' concentrations (and their respective ratios) as the reaction goes on. It is known for DAIB-catalysed reactions that the reactants and, in certain cases, the reaction products have an influence on the catalyst composition, which results in altered reaction rates and non-constant ee $e_{P}$ over time. ${ }^{9,29}$ Indeed, an 
increase of $e_{\mathrm{p}}$ over the course of the reaction was observed in the NBE-catalysed reaction. ${ }^{11}$ Therefore, further studies (and quantitative analyses in particular) of NME- and NBE-catalysed dialkylzinc additions will require more complex models, which include the concentrations of the starting materials and of the products and their possible influence on the catalyst distribution.

\section{Conclusion}

In summary, we have found an enantiodivergent behaviour in NME-catalysed reactions which may be explained by the monomer-dimer competition model, known for the parent ligand NBE. Simulation allowed us to confirm this and to get further insights into the model, such as the dependence of the observed kinetic constant $\mathrm{k}_{\mathrm{obs}}$ on the catalyst concentration and on the enantiomeric excess of the ligand. We expect this may serve as a basis for further research to understand past and future cases of enantiodivergent non-linear effects, as well as of asymmetric catalytic systems in general. Further work in our group will aim at the elucidation and the quantification of the different parameters governing reactions catalysed by ephedrine-derivatives, at the extension of the models in order to include the influence of reactants and products and at the investigation of aggregation-induced complex catalytic systems in a general way.

\section{Data availability}

Synthetic and mathematical procedures, as well as additional data and chromatograms for every experimental catalytic run can be found in the Supplementary Information. Detailed experimental data (reactant quantities, reaction conditions, raw and treated results for all catalytic runs, including reactions with NBE) and calculated data for all simulated graphs in this paper can be found in the respective excel files (SI_experimental_Data.xlsx, SI_Simulated_NLE.xlsx, SI_kinetic_model.xlsx).

\section{Author contributions}

Y.G. performed the synthetic experiments and developed the catalytic models, along with their mathematical expressions. A.M.-F. and T.A. participated in data analyses. S.B.-L. conceptualized and supervised the study and wrote the manuscript with Y.G. 


\section{Conflicts of interest}

There are no conflicts to declare.

\section{References}

1 H. B. Kagan and T. O. Luukas, in Comprehensive Asymmetric Catalysis, eds. E. N. Jacobsen, A. Pfaltz and H. Yamamoto, Springer-Verlag, Berlin, 1999, vol. I, pp. 101-118.

2 C. Puchot, O. Samuel, E. Dunach, S. Zhao, C. Agami and H. B. Kagan, Nonlinear effects in asymmetric synthesis. Examples in asymmetric oxidations and aldolization reactions, J. Am. Chem. Soc., 1986, 108, 2353-2357.

3 H. B. Kagan, Nonlinear Effects in Enantioselective Organometallic Catalysis, Oil Gas Sci. Technol., 2007, 62, 731-738.

4 C. Girard and H. B. Kagan, Nonlinear Effects in Asymmetric Synthesis and Stereoselective Reactions: Ten Years of Investigation, Angew. Chem. Int. Ed., 1998, 37, 2922-2959.

5 T. Satyanarayana, S. Abraham and H. B. Kagan, Nonlinear Effects in Asymmetric Catalysis, Angew. Chem. Int. Ed., 2009, 48, 456-494.

6 D. G. Blackmond, The Origin of Biological Homochirality, Cold Spring Harbor Perspect. Biol., 2019, 11, a032540.

7 D. Guillaneux, S.-H. Zhao, O. Samuel, D. Rainford and H. B. Kagan, Nonlinear effects in asymmetric catalysis, J. Am. Chem. Soc., 1994, 116, 9430-9439.

8 M. Kitamura, S. Okada, S. Suga and R. Noyori, Enantioselective addition of dialkylzincs to aldehydes promoted by chiral amino alcohols. Mechanism and nonlinear effect, J. Am. Chem. Soc., 1989, 111, 4028-4036.

9 M. Kitamura, S. Suga, H. Oka and R. Noyori, Quantitative Analysis of the Chiral Amplification in the Amino Alcohol-Promoted Asymmetric Alkylation of Aldehydes with Dialkylzincs, J. Am. Chem. Soc., 1998, 120, 9800-9809.

10 M. Tsukamoto, K. Gopalaiah and H. B. Kagan, Equilibrium of Homochiral Oligomerization of a Mixture of Enantiomers. Its Relevance to Nonlinear Effects in Asymmetric Catalysis, J. Phys. Chem. B, 2008, 112, 15361-15368.

11 Y. Geiger, T. Achard, A. Maisse-François and S. Bellemin-Laponnaz, Hyperpositive nonlinear effects in asymmetric catalysis, Nat. Catal., 2020, 3, 422-426.

12 Y. Geiger, T. Achard, A. Maisse-François and S. Bellemin-Laponnaz, Observation of hyperpositive nonlinear effect in catalytic asymmetric organozinc additions to aldehydes, Chirality, , DOI:10.1002/chir.23271.

13 R. Noyori, S. Suga, H. Oka and M. Kitamura, Self and nonself recognition of chiral catalysts: The origin of nonlinear effects in the amino-alcohol catalyzed asymmetric addition of diorganozincs to aldehydes, Chem. Record., 2001, 1, 85-100.

14 A. Cruz, I. Irene Padilla-Martínez and M. Esther Bautista-Ramirez, N-Substituted Ephedrines as Chiral Auxiliaries in Enantioselective Alkylation Reactions of Carbonyl Compounds, Curr. Org. Synth., 2016, 13, 2-40.

15 C. Muchow, Y. Vannoorenberghe and G. Buono, Use of alkaloids and aminoalcohols in catalytic asymmetric induction:Temperature effect on the addition of diethylzinc to benzaldehyde, Tetrahedron Lett., 1987, 28, 6163-6166. 
16 P. A. Chaloner, E. Langadianou and S. A. R. Perera, Enantioselective reaction of diethylzinc with arenecarbaldehydes in the presence of ephedrine derivatives, J. Chem. Soc., Perkin Trans. 1, 1991, 0, 2731-2735.

17 K. Soai, S. Yokohama and T. Hayasaka, Chiral N,N-Dialkylnorephedrines as Catalysts of the Highly Enantioselective Addition of Dialkylzincs to Aliphatic and Aromatic Aldehydes. The Asymmetric Synthesis of Secondary Aliphatic and Aromatic Alcohols of High Optical Purity, J. Org. Chem., 1991, 56, 4264-4268.

18 K. Fitzpatrick, R. Hulst and R. M. Kellogg, Thiol and disulfide derivatives of ephedra alkaloids 2: A mechanistic study of their effect on the addition of diethyl zinc to benzaldehyde, Tetrahedron: Asymmetry, 1995, 6, 1861-1864.

19 M. Sibi and M. Liu, Reversal of Stereochemistry in Enantioselective Transformations. Can they be Planned or are they Just Accidental?, Curr. Org. Chem., 2001, 5, 719-755.

$20 \mathrm{~J}$. Escorihuela, M. I. Burguete and S. V. Luis, New advances in dual stereocontrol for asymmetric reactions, Chem. Soc. Rev., 2013, 42, 5595-5617.

21 I. P. Beletskaya, C. Nájera and M. Yus, Stereodivergent Catalysis, Chem. Rev., 2018, 118, 5080-5200.

22 W. Cao, X. Feng and X. Liu, Reversal of enantioselectivity in chiral metal complex-catalyzed asymmetric reactions, Org. Biomol. Chem., 2019, 17, 6538-6550.

23 The effect of catalyst concentration has been studied in one case, however it was the ligand-to-metal ratio which turned out to be the key factor to enantiodivergence there, $c f$. A. M. Porte, J. Reibenspies and K. Burgess, Design and Optimization of New Phosphine Oxazoline Ligands via High-Throughput Catalyst Screening, J. Am. Chem. Soc., 1998, 120, 9180-9187., .

$24 \mathrm{H}$. Du, J. Long, J. Hu, X. Li and K. Ding, 3,3'-Br2-BINOL-Zn Complex: A Highly Efficient Catalyst for the Enantioselective Hetero-Diels-Alder Reaction, Org. Lett., 2002, 4, 4349-4352.

$25 \mathrm{H}$. Du, X. Zhang, Z. Wang and K. Ding, One catalyst for two distinct reactions: sequential asymmetric hetero Diels-Alder reaction and diethylzinc addition, Tetrahedron, 2005, 61, 9465-9477.

$26 \mathrm{P}$. Wipf, N. Jayasuriya and S. Ribe, On the role of chiral catalysts in the alkenyl zirconocene/zinc addition to aldehydes: A study of ligand loading and asymmetric amplification, Chirality, 2003, 15, 208-212.

27 Only Kagan mentioned this briefly when discussing the general $M L_{n}$ model (cf. ref. 7).

28 F. Buono, P. J. Walsh and D. G. Blackmond, Rationalization of Anomalous Nonlinear Effects in the Alkylation of Substituted Benzaldehydes, J. Am. Chem. Soc., 2002, 124, 13652-13653.

29 T. Rosner, P. J. Sears, W. A. Nugent and D. G. Blackmond, Kinetic Investigations of Product Inhibition in the Amino Alcohol-Catalyzed Asymmetric Alkylation of Benzaldehyde with Diethylzinc, Org. Lett., 2000, 2, 2511-2513.

30 Noble-Terán and co-workers discussed that possibility, but built their kinetic models for the two extreme cases were either only monomers or dimers catalyse, cf. M. E. Noble-Terán, T. Buhse, J.-M. Cruz, C. Coudret and J.-C. Micheau, Nonlinear Effects in Asymmetric Synthesis: A Practical Tool for the Discrimination between Monomer and Dimer Catalysis, ChemCatChem, 2016, 8, 1836-1845.

$31 K_{\text {Homo }}$ in Models I and II should not be confused with Noyori's $K_{\text {homo, }}$ which was defined as a dissociation constant (cf. ref. 9).

32 M. Kitamura, S. Suga, M. Niwa, R. Noyori, Z.-X. Zhai and H. Suga, Enantiomer Recognition of Asymmetric Catalysts. Thermodynamic Properties of Homochiral and Heterochiral Dimers of the Methylzinc Alkoxide Formed from Dimethylzinc and Enantiomeric 3-exo-(Dimethylamino)isoborneol, J. Phys. Chem., 1994, 98, 12776-12781.

33 D. G. Blackmond, Mathematical Models of Nonlinear Effects in Asymmetric Catalysis: New Insights Based on the Role of Reaction Rate, J. Am. Chem. Soc., 1997, 119, 12934-12939.

34 D. G. Blackmond, Kinetic aspects of non-linear effects in asymmetric synthesis, catalysis, and autocatalysis, Tetrahedron: Asymmetry, 2010, 21, 1630-1634. 
35 M. E. Noble-Terán, T. Buhse, J.-M. Cruz, C. Coudret and J.-C. Micheau, Nonlinear Effects in Asymmetric Synthesis: A Practical Tool for the Discrimination between Monomer and Dimer Catalysis, ChemCatChem, 2016, 8, 1836-1845.

$36 \mathrm{~J} .-$ C. Micheau, T. Buhse, D. Lavabre and J. R. Islas, Kinetic understanding of asymmetric amplification in amino-alcohol catalyzed organozinc additions, Tetrahedron: Asymmetry, 2008, 19, 416-424.

37 J. Burés, A Simple Graphical Method to Determine the Order in Catalyst, Angew. Chem. Int. Ed., 2016, 55, 2028-2031.

38 J. Burés, Variable Time Normalization Analysis: General Graphical Elucidation of Reaction Orders from Concentration Profiles, Angew. Chem. Int. Ed., 2016, 55, 16084-16087.

39 C. D.-T. Nielsen and J. Burés, Visual kinetic analysis, Chem. Sci., 2019, 10, 348-353.

40 J. Burés, What is the Order of a Reaction?, Top. Catal., 2017, 60, 631-633.

41 A. M. Porte, J. Reibenspies and K. Burgess, Design and Optimization of New Phosphine Oxazoline Ligands via High-Throughput Catalyst Screening, J. Am. Chem. Soc., 1998, 120, 9180-9187.

42 B. Schmidt and D. Seebach, Katalytische und stöchiometrische enantioselektive Additionen von Diethylzink an Aldehyde mit Hilfe eines neuartigen chiralen Spirotitanats, Angew. Chem., 1991, 103, 100-101.

43 B. Schmidt and D. Seebach, 2,2-Dimethyl- $\alpha, \alpha, \alpha^{\prime}, \alpha^{\prime}$-tetra(naphth-2-yl)-1,3-dioxolan-4,5-dimethanol (DINOL) für die Titanat-vermittelte, enantioselektive Addition von Diethylzink an Aldehyde, Angew. Chem., 1991, 103, 1383-1385.

44 D. Seebach, D. A. Plattner, A. K. Beck, Y. M. Wang, D. Hunziker and W. Petter, On the Mechanisms of Enantioselective Reactions Using $\alpha, \alpha, \alpha^{\prime}, \alpha^{\prime}$-Tetraaryl-1,3-dioxolane-4,5-dimethanol(TADDOL)-Derived Titanates: Differences between C2- and C1-symmetrical TADDOLs - facts, implications and generalizations, Helv. Chim. Acta, 1992, 75, 2171-2209.

45 H. Danjo, M. Higuchi, M. Yada and T. Imamoto, P-stereogenic P/N hybrid ligands: a remarkable switch in enantioselectivity in palladium-catalyzed asymmetric allylation, Tetrahedron Lett., 2004, 45, 603606.

46Z. Shao, J. Wang, K. Ding and A. S. C. Chan, Unprecedented Effects of Additives and Ligand-to-Metal Ratio on the Enantiofacial Selection of Copper-Catalyzed Alkynylation of $\alpha$-Imino Ester with Arylacetylenes, Adv. Synth. Catal., 2007, 349, 2375-2379.

47 F. Peng, Z. Shao and A. S. C. Chan, Copper(I)-catalyzed enantioselective alkynylation of $\alpha$-imino esters: ligand-to-metal ratio effects and mechanistic studies, Tetrahedron: Asymmetry, 2010, 21, 465-468.

48 M. I. Burguete, M. Collado, J. Escorihuela and S. V. Luis, Efficient Chirality Switching in the Addition of Diethylzinc to Aldehydes in the Presence of Simple Chiral a-Amino Amides, Angew. Chem. Int. Ed., 2007, 46, 9002-9005.

49 J. Li, S.-S. Wang, P.-J. Xia, Q.-L. Zhao, J.-A. Xiao, H.-Y. Xiang, X.-Q. Chen and H. Yang, Unusual Ligand-toMetal-Ratio-Controlled Bidirectional Enantioselectivity in Pd-Catalysed [3+3]-Annulation of MoritaBaylis-Hillman Acetate, Eur. J. Org. Chem., 2017, 2017, 6961-6965. 